\title{
Medios de comunicación en Afganistán: la fragilidad de la explosión mediática en la última década (2002-2012)
}

\author{
Alejandra VAL CUBERo \\ Universidad Carlos III de Madrid \\ Alejandra.val@uc3m.es
}

Recibido: $16 / 11 / 2013$

Aceptado: 22/02/2013

\section{Resumen}

El análisis de los medios de comunicación en Afganistán presenta un desafío para todos los estudios en este ámbito, al ser un país con una larga historia periodística y una corta historia televisiva. La explosión mediática acontecida desde la llegada de las tropas extranjeras en el año 2001 alteró el mapa comunicativo con la implementación de nuevas cadenas televisivas, radios y proveedores de internet. Los organismos internacionales a través de ingentes donaciones trataron de crear medios independientes que fortalecieran el sistema democrático y contrarrestaran las comunicaciones de los insurgentes. Pretensiones que hoy están en el aire porque los principales medios dependen de la ayuda externa, en una población que sigue sin confiar en ellos y en un momento en el que crecen las informaciones de los movimientos más extremistas.

Palabras clave: Afganistán, medios de comunicación, desarrollo.

Media in Afghanistan:

the fragility of the media explosion in the past decade (2002-2012)

\begin{abstract}
The analysis of media in Afghanistan represents a challenge for all studies in this area, being a country with a long jounalistic history and a short television history. The media explosion which occurred since the arrival of foreign troops in 2001 altered the communication map with the arrival of new network television, radio and internet providers. International agencies through huge donations tried to create independent media that would strengthen the democratic system and counteract insurgents communications. A target that is still to be reached since most media outlets depend on foreign aid, in a population that still does not trust them and at a time of increasing information provided by extremist movements.
\end{abstract}

Keywords: Afganistán, medios de comunicación, desarrollo.

\section{Referencia normalizada}

VAL CUBERO, Alejandra (2013): "Medios de comunicación en Afganistán: la fragilidad de la explosión mediática en la última década (2002-2012)". Estudios sobre el Mensaje Periodístico. Vol. 19, Núm. 2 (julio-diciembre), págs.: 907-919. Madrid, Servicio de Publicaciones de la Universidad Complutense.

Sumario: 1. Introducción 2. Sistema Audiovisual Afgano 3. Prensa: un largo recorrido de futuro incierto. 4. Radio: el medio más extendido. 5. Televisión: la ventana al entretenimiento. 6. Programas televisivos: de la religión al juego. 7. A modo de conclusión. 8. Referencias; 8.1. Webs consultadas.

\section{Introducción}

El estudio de los medios de comunicación en Afganistán desde el año 2001 presenta un caso de interés para los investigadores en comunicación por su rápido desarrollo. En sólo una década el número de medios se ha multiplicado exponencialmente pasando de una decena de radios a más de cien a finales del 2010 (Altai, 2010: 50). 
La breve historia de los medios de comunicación en Afganistán está ligada a periodos de progreso económico y social como el que se produjo bajo el mandato del rey Amanullah Khan entre 1919 y 1929. Khan promulgó ciertas reformas que dieron pie a la aprobación de la primera Constitución afgana bajo la idea de que uno de los principales requisitos de avance social pasaban por instaurar un sistema escolar universal y gratuito y proteger la libertad de prensa. En estos años un total de veintitrés periódicos y revistas salieron a la luz en Kabul y en otras ciudades afganas (Grevemeyer, 1987: 99), incluido el primer periódico de mujeres Erschad-al-Nasswan-Recomendaciones para las Mujeres- publicado por la reina Soraja en 1922 (Yussufi, 1977: 92). Tras su muerte y durante el reinado de Nadir Shah's (1929-1933) y de su hijo Zahir Shah (1933-1973) las políticas comunicativas estuvieron definidas en virtud de dos características: impulsar la conciencia nacional y poner énfasis en la identidad histórica y cultural. La primera agencia afgana de noticias Bakhtar creada en 1939 y la puesta en marcha de Radio Kabul en 1940 fueron los dos pilares en los que se apoyó esta estrategia mediática.

Entre 1963 y 1973 bajo el mandato del rey Zahir Shah Afganistán gozó de una libertad sin precedentes promulgando el voto femenino. El rey impulsó los estudios de periodismo y creó el Modiriat-e Konferenzha -Departamento de conferencias- en 1964 para facilitar las conexiones informativas entre la capital y el resto de las regiones (Grevemeyer, 1987: 316). Tras finalizar su mandato había setenta diarios, revistas y otras publicaciones de las cuales dieciséis eran periódicos de tirada diaria. El golpe militar de Mohammad Daud Khan en 1973 y la proclamación de la República de Afganistán no alteró el mapa mediático aunque la censura fue mayor. Sin embargo el sistema mediático colapsó tras la toma del poder del líder pro-soviético Najibullah en 1992 (Rawan, 2002). En los cuatro años que estuvo en el poder sólo unos pocos medios permanecieron en la escena pública y los que lo hicieron tenían un marcado perfil político. Los periódicos nacionales Anis, Hewad y The Kabul News Times se siguieron publicando con calidad era muy modesta, ofreciendo una información muy sesgada al no permitirse la publicación de ningún artículo crítico con el dirigente y su gobierno.

Los medios de comunicación estuvieron prohibidos bajo el mandato talibán (19962001)1. La televisión fue censurada y Radio Afghanistan pasó a llamarse Radio Voice of Shari'a que recuperaría su antiguo nombre el 13 de noviembre de 2002, el mismo año en el que aparecería en escena Kabul TV. A partir de esta fecha el crecimiento televisivo y radiofónico ha sido exponencial tanto a nivel nacional como regional o local. En el año 2005 ya estaban funcionando cuarenta y dos estaciones de radio y ocho estaciones televisivas (Altai, 2005: 86). Y a comienzos del 2006 hicieron su presencia las cadenas televisivas y radios regionales y locales. En Mazar i- Sharif capi-

1 Durante el periodo talibán el único medio de comunicación era Radio Shari'a y algunas publicaciones religiosas gubernamentales. La televisión estaba prohibida y el servicio telefónico era tan malo que las personas que podían permitirse realizar una llamada al extranjero debían viajar a Pakistán. 
tal de la provincia de Balkh al norte del país surgieron Arezo TV (2007), Setara-eSahar TV (2010) y las radios Nehad y Omid-e-Jawan (2006), Lahza (2008), Shahar (2009), Sabz, Mehraban y Band (2010). En la región sureña de Kandahar se creó Hewad TV y las cadenas de radio Killid (2007), Talimul Islam y Wranga (2009). En Jalalabad al este del país y frontera con Pakistán se puso en marcha Nangarhar RTA (2006), Sharq TV (2008) y las radios Muram (2008), Abasin, Safa, Narghes, Killid (2009) y Menbar (2010). Y en Herat al oeste del país y frontera con Irán se crearon las cadenas televisivas Herai (2007), Taban (2008), Asia y Mayhan (2010) y las estaciones de radio Faryad (2006), Watandar Herat (2007), Shindand, Baran y Zohal (2008).

Este desarrollo mediático sin precedentes en el país ha sido posible a la confluencia de una serie de factores políticos, económicos y jurídicos: por una parte la elevada atención de Estados Unidos y Europa principalmente y sus ayudas al sector de la comunicación, y por otra el impulso del gobierno afgano con la creación de varios consejos consultativos y la aprobación de una Ley de Medios en el año 2002.

Una de las primeras organizaciones no gubernamentales en prestar sus servicios en Afganistán fue Internews que con su lema local voices, global change ha sido la promotora en crear y gestionar radios independientes, así como en formar a periodistas afganos ${ }^{2}$. La Unesco apoyó al sector de los medios impresos con la salida de Kabul Weekly y Roz Magazine y dirigió The Afghan Media and Culture Center creada por la ong Aïna en el año 2002. Y la Agencia para el Desarrollo Americana (USAID) invirtió en la infraestructura de lo que sería la cadena de radio Arman FM en el año 2003, financió la compra de transmisores de la cadena televisiva Tolo $T V$ en 2004, e impulsó la existencia del Afghan Media Development (AMD) que en los primeros dos años de existencia (2002-2004) creó treinta estaciones de radio, más de docientos periódicos y revistas y cuatro cadenas de televisión. En estos años también apareció la agencia de noticias PAN bajo el patronazgo del Institute for War \& Peace Reporting (IWPR) (2002) financiada por las donaciones del gobierno Noruego (60-70\% de los fondos), Suecia y Estados Unidos y que ha tratado de jugar un papel de liderazgo en la profesionalización de los medios de comunicación tal y como explican en su web ${ }^{3}$. Estos son sólo algunos ejemplos del interés que los países aliados han puesto para desarrollar el mapa audiovisual en Afganistán, es innegable que se han creado nuevos medios pero la manera en la que se ha llevado a cabo, su influencia y su posterior desarrollo es algo que aún está por definir y que configuran un sistema audiovisual frágil.

2 Internews: http://www.internews.org/. Ä̈na e Internews crearon la productora Awaz en el año 2004 y produce programas sobre temas sociales (salud, educación, derechos políticos). Uno de sus programas estelares ha sido "Facing the Nation" emitido en la RTA (Radio Televisión en Afganistán) como plataforma para que los líderes políticos expusieran y defendieran sus programas políticos y en los que eran interrogados por los espectadores. Awaz trabaja con donantes internacionales y con medios de comunicación nacionales como Ariana $T V$, RTA, Lemar y Shamshad y en menor medida Tolo TV.

3 Institute for War \& Peace Reporting (IWPR): http://iwpr.net/ 


\section{Sistema audiovisual afgano}

La Constitución aprobada por la Loya Jirga (Gran Asamblea) en enero de 2004 expone que la Ley de Medios tiene que estar regulada por una legislación especial no recogida en dicho texto. La Constitución incluye una serie de artículos para el correcto funcionamiento de los medios, como la libertad de expresión y el derecho de los medios para publicar informaciones sin la autorización gubernamental, tal y como lo presenta en su artículo 34 aunque esta ley contrasta con el artículo 45, donde se menciona que hay ciertos temas que están prohibidos por la Constitución: lo que resulte contrario a los principios del Islám, lo que resulte difamatorio, ofensivo o insultante para el gobierno, o las informaciones que sean contrarias a la Ley Criminal ${ }^{4}$.

La primera Ley de Medios en Afganistán fue aprobada en marzo de 2002 por la $\mathrm{Wo}_{\text {- }}$ lesi Jirga (o Cámara Baja del Parlamento) destaca en su artículo segundo la intención de promover y apoyar el derecho de libertad de pensamiento y de palabra, defender los derechos de los periodistas, promover y desarrollar medios de comunicación pluralistas, independientes y libres y procurar y facilitar un contexto que sea adecuado para la libre expresión de los ciudadanos ${ }^{5}$. La ley establece dos órganos de supervisión, el Mass Media Commission (MMC) formado por siete profesionales del periodismo y un órgano judicial superior que es el High Media Council (HMC) compuesto por trece miembros, tres del Ministerio de Cultura, Justicia y Comunicación, uno de la Corte Suprema, cuatro de las dos Cámaras del Parlamento, un representante religioso, dos miembros de la comunidad mediática y dos de la civil.

Otros órganos destacados en el correcto funcionamiento de los medios son el Ministerio de Información y Cultura (MoIC) encargado de dar licencias a las radios y televisiones y gestionar los medios públicos y el Media and Information Center (GMIC) ${ }^{6}$. GMIC es una organización creada por el Presidente Karzai en 2008 con el objetivo de crear puentes entre el gobierno y la ciudadanía y el gobierno y la comunidad internacional. El GMIC trabaja principalmente en tres áreas: la coordinación y las relaciones con los distintos medios -organiza conferencias entre el MoIC, los representantes en las embajadas, o la Fuerza Internacional para la Asistencia Internacional (ISAF)-, prepara boletines e informes para el Presidente y sus ministros, crea cursos de capacitación y entrenamiento para los portavoces de Gobierno y trabaja en los medios tradicionales (carteles, boletines ...) para transmitir mensajes públicos a la población rural a través de los grupos de ancianos o representantes de cada comunidad (Unesco, 2012).

4 "Freedom of expression is inviolable. Every Afghan has the right to express his thought through speech, writing, illustration or other means, by observing the provisions stated in this Constitution. Every Afghan has the right to print or publish material without prior submission to the state authorities in accordance with law. Provisions related to printing houses, radio, television, press, and other mass media, will be regulated by law". Art. 34 de la Constitución Afgana. Traducción propia

5 La Ley de Medios en Afganistán ha sido modificada en tres ocasiones: en abril de 2004, en junio 2006 y en agosto de 2008 .

6 Ministerio de Información y Cultura (MoIC): http://moic.gov.af/en 


\section{Prensa: un largo recorrido de futuro incierto}

La mayoría de los medios impresos -periódicos, revistas o semanarios- afganos reciben ayudas para su publicación y distribución. Kabul Weekly el primer periódico independiente en salir a circulación después de la caída del régimen talibán en enero de 2002 comenzó su andadura en el año 1991, pero su publicación estuvo interrumpida de 1994 a 2002. Kabul Weekly recibe subvención de la Unesco, tiene una circulación de 10,000 a 15,000 ejemplares, sus artículos están en inglés, dari y pastu y cuenta con una versión online desde el año 20087. Los medios más longevos como Anis (1927), Hewad (1949) y The Kabul Times (1962) cuentan con una circulación más limitada (menor de 5000 ejemplares) y sus editoriales apoyan la agenda del gobierno, al igual que sucede con la revista gratuita Sada-e-Azadi publicada por la Fuerza Internacional de Asistencia para la Seguridad ISAF y con una distribución de medio millón de copias en todo el país ${ }^{8}$.

La mayoría de los medios impresos defienden en su artículos las actividades y decisiones del gobierno de Karzai y no son muy críticos con la presencia de las tropas extranjeras. Sin embargo hay medios que no se alían con el partido en el poder y defienden otros intereses políticos o sociales, entre ellos Hasht Sobh (8am) creado en el año 2006 y muy comprometido con todos los temas que atañen a las minorías raciales en Afganistán ${ }^{9}$ y Mandegar (Permanente) con una circulación de 12.000 copias y un posicionamiento muy crítico con el gobierno. Mandegar nació el año 2009 en apoyo de Abdulá Abdulá, líder de la oposición durante las elecciones de este mismo año y permanece en escena gracias a las donaciones de los ciudadanos que apoyaron su candidatura.

Hay otros periódicos y semanarios que reflejan la diversidad de tendencias políticas del país: Cheragh (La Lámpara) apoya al partido Jamiat Islami; Mardom (Gente) es la publicación oficial del partido Harakat Elami del Ayatollah Assef Mohseni; Weesa es una publicación de los nacionalistas pastunes; o Afghanistan (y su versión en inglés Outlook) relacionada con el partido Wahdat Islami -el partido de Mohammad Mohaqiq. Entre las revistas las más leídas Killid ofrece cada semana cuarenta páginas de artículos en dari, pastú e inglés sobre temas que van desde la política, deportes o el cine y Morsal un semanario parecido pero de menor tamaño (30 páginas) más dirigido al público femenino y que trata temas sociales, de salud, o familia.

La prensa en Afganistán pese a ser el medio con una historia lonjeva es el que menos ha crecido en la última década (2003-2013) si lo comparamos con otros medios como la radio o la televisión. Hay varios factores que han limitado su desarrollo y que ponen en peligro su continuidad. La alta tasa de analfabetismo entre la población afgana donde sólo un $43 \%$ hombres saben leer frente al 12,6\% de las mujeres (The World FactBook, 2012) y la pobreza en la que vive la mayoría de la población hace que el medio impreso sea contemplado como un objeto de lujo. Al tiempo que

7 Kabul Weekly: www.kw.af.

8 Los artículos también aparecen en los tres idiomas dari, pastú e inglés. Web de la revista Sada-e-Azadi: http://www.sada-e-azadi.net/index.php/en/print

9 Hast Sobh tiene una tirada de 15.000 copias. 
la compleja holografía del país dificulta aún más su distribución e impide que muchos periódicos y revistas lleguen a su destino.

El número de agencias de noticias también ha crecido considerablemente y desde el año 2001 existen once agencias de noticias la mayoría con sede en Kabul. La agencia gubernamental Bakhtar y las privadas Pajhwok Afghan News (PAN), Roz, Hindu Kush, Wact y Afghan Islamic Press (con sede en Pakistan). Pajhwok Afghan News (PAN) es la agencia de noticias líder creada en el año 2004 y subvencionado por USAID (18\%) y la Open Society Institute (OSI) (20\%). PAN tiene cuarenta y cinco reporteros a lo largo del país y quince periodistas freelance que trabajan en el exterior y entre sus suscritores se incluyen trece periódicos nacionales y diez internacionales, sesenta cadenas de radio nacionales y diez internacionales y diecisiete cadenas de televisión y el precio de la inscripción mensual varía entre 300 a 400 dólares al mes ${ }^{10}$.

La llegada y el desarrollo de internet han permitido la creación de webs informativas escritas en su mayoría en inglés y leídas por un público joven con estudios universitarios. Benawa (2004) se define como la web online de la comunidad pastú. Las informaciones provienen de voluntarios residentes dentro y fuera de Afganistán, principalmente en Pakistán, Irán y Europa ${ }^{11}$. Payam-e-Aftab (El mensaje del sol) con contenidos en inglés, dari y pastú es una web informativa persa fundada por Seyed Mohammad Baqer, con informaciones políticas nacionales e internacionales y ensayos sobre religión y cultura que cuenta con el sustento de los movimientos islamistas moderados $^{12}$. Kabul Press es una de las web más críticas con el gobierno. La página ha sido acusada de ser de ser pro-pastún y cerrada por el gobierno en varias ocasiones al estar relacionada con los talibanes ${ }^{13}$.

\section{Radio: el medio más extendido}

Hasta hace pocos años la radio era el único medio de comunicación en Afganistán. En la actualidad el número de radios en el país es muy elevado (más de ciento cincuenta en septiembre de 2010) y la mayoría de cadenas de radio están asociadas a cadenas televisivas. Hay varios tipos de cadenas de radio: internacionales como la $B B C, V O A$, Azadi, Sada-e-Azadi, cadenas privadas nacionales Arman, Killid, Sharq, Sol-e-Paygham, cadenas comunitarias con pocos empleados y pequeñas cadenas de radio.

Entre las cadenas internacionales $B B C$ comenzó a emitir en el año 1980 y cuenta en la actualidad con veinte estaciones de FM y es accesible en AM en casi todas las regiones del país. Las noticias internacionales proceden de Londres y el resto de la programación se produce en Afganistán. Azadi creada en el año 2003 emite doce horas al día (7am-7pm) comparte la misma frecuencia que Ashna/VOA (que cubre el resto del día (7pm-7am) y cuenta con una estación de FM en las cinco mayores ciudades de Afganistán: Kabul, Jalalabad, Mazar-e-Sharif, Kandahar and Herat y sus programas se centran principalmente en mostrar la cultura y la identidad afgana. El $40 \%$ del con-

\footnotetext{
${ }^{10}$ Pajhwok Afghan News (PAN), http://www.pajhwok.com/

${ }^{11}$ Benawa: www.benawa.com

${ }^{12}$ Payam-aftab: www.payam-aftab.com

${ }^{13}$ Kabul Press: www.kabulpress.org
} 
tenido se produce en la oficina central en Praga. Ashna/VOA tiene cinco estaciones FM en Kabul, Jalalabad, Mazar-e-Sharif, Kandahar y Herat y se puede oir en Irán, la cadena emite programas en dari y pastu. El 100\% de la programación se decide en Washington y es una cadena pro-americana. Sada-e-Azadi (La voz de la libertad) es la cadena del ISAF que emite en dari y pastú. La mayor parte de su programación se centra en la música, los concursos y las entrevistas y defiende los intereses de las tropas internacionales.

Las radios estatales como Radio Afganistán tienen como objetivo promover el arte y la cultura (principalmente la música) afgana e informar a la población sobre temas como los procesos electorales y los cambios político aunque la principal radio cultural afgana es Killid con programas dedicados a poetas afganos, música afgana, india o pakistaní y cantantes tradicionales. Durante varios años solo emitió en Kabul pero ahora tiene sedes en las principales ciudades afganas: Herat, Mazar-e-Sharif, Kandahar, Jalalabad. Arman es otra de las cadenas a nivel nacional con mayor audiencia, emite música occidental y el lenguaje es más coloquial que otras cadenas de estas características ${ }^{14}$.

\section{La Television: la ventana al entretenimiento}

La historia de la televisión contemporánea es mucho más reciente que la historia de la prensa y se puede estudiar a partir del año 2001. En Afganistán existen cadenas públicas, privadas, regionales y locales, cada una con un perfil en función de su fundador, financiación y zona geográfica en la que se emita.

Ariana $T V$ creada en el 2005 es la cadena con mayor mayor índice de audiencia y mayor cobertura, -llega a treinta y tres de las treinta y cuatro provincias afganas con excepción de la región del Nuristan, al este del país-. Ariana TV es una cadena centrista, no tiene un tono conservador o religiosa pero tampoco liberal y aunque no se define políticamente suele apoyar al Presidente Karzai y a su administración. El Presidente de la cadena Ehsan Bayat es también el fundador del Afghan Wireless Communication Company (AWCC) y The Bayat Foundation Charity. Sus programas son principalmente en dari (45\%), pastu (35\%), inglés $(5 \%)$ y Uzbeco $(15 \%)^{15}$. La cadena privada Tolo $T V$ fundada en el año 2004 pertenece a la familia Mohseni que también gestiona el grupo de comunicación Moby Group que engloba Tolo Arman Radio, Lemar y la cadena por satélite Tolo News, la compañía de producción Kaboora, la agencia de publicidad Lapis, y la revista Afghan Scene, entre otros medios. La segunda cadena con más audiencia Tolo TV apoya al Presidente Karzai, al igual que Yak $T V$, otra de las cadenas privadas en línea con las actividades de la North Atlantic Treaty Organization (NATO) y del gobierno, o Saba TV que emite las veinticuatro horas en nueve provincias, pertenece al grupo Saba Group propietaria de la estación de radio (Nawa), grupo comunicativo centrado en los programas educativos cuyos trabajadores provienen del Afghan Independent Human Rights Commission ${ }^{16}$.

\footnotetext{
${ }^{14}$ Las cadenas de radio más populares son la cadena pública Radio Afghanistan, Balkh Radio, Radio Kabul, Radio Heart y Voice of Freedom.

${ }^{15}$ Ariana TV: http://arianatelevision.com/

${ }^{16}$ Tolo TV: http://tolo.tv/
} 
Entre los medios públicos está la Radio Television Afganistán (RTA) financiada por el Ministerio de Información y Cultura. RTA es la tercera cadena en audiencia después de Tolo TV y Ariadna. Llega a todas las provincias y su objetivo es promover el sentimiento de unidad nacional y la responsabilidad civil, con programas como Dast Award (Logro), Ayenda Sazan (Creadores de Futuro) y Rahi Be Suye Khushbakhti (El camino hacia la felicidad).

La mayoría de las cadenas generalistas emiten en los dos idiomas oficiales del país (el dari y el pastú) pero hay cadenas que priorizan uno de los dos idiomas o incluso un idioma minoritario como el uzbeco. Shamshad TV es una cadena con contenidos exclusivamente en lengua pastú, sus programas provienen de Pakistán o Medio Oriente y una gran proporción se traducen y adaptan. El tono de la cadena es liberal y apoyan el movimiento nacionalista pastú. Lemar es otra de las cadenas de Moby Group y emita en pastú convirtiéndose en la cadena con mayor audiencia en las provincias del sur y sureste del país com Kandahar, Helmand, Daykundi, Farah, Uruzgan ${ }^{17}$.

Desde el 2006 han aparecido a escena pública cadenas televisivas financiadas por organismos religiosos y políticos. Entre las cadenas religiosas están Kawsar (nombre del versículo 108 del Corán), Tamaddon (Civilización) y Da'wat (Predicación). Las cadenas se financian con fondos privados, no existe publicidad y no suelen aceptar fondos gubernamentales o de la comunidad internacional. Tamaddon es el más conocida bajo el slogan: "El ideal de la mayoría silenciosa", lanzada por el Ayatollah Asef Mohseni líder del movimiento Harakat-e-Islami y fundador de la universidad religiosa Khatem al-Nabiin en Kabul. Los programas que emite son moderados y próximos a Irán. Kawsar es una televisión más beligerante y en el acto de inauguración por el Ayatollah Taqadossi en la madrassa de Madinat al-Alam (Kabul) el 12 de mayo de 2006 señaló que La cadena de televisión Kawsar nacía para oponerse a la invasión cultural occidental y enseñar los principios de la religión sagrada del Islám.

Entre los medios de comunicación ligados a distintos partidos políticos está la cadena televisiva próxima a Irán Noor (Luz) (2008) que representa los intereses del partido Jamiat-e-Islami con contenidos críticos con Pakistán. Otras cadenas políticas son Rah-e-Farda creada por Mohammed Mohaqiq, líder de Hezb-e-Wahdat. Negah (dirigida por el hijo del Vice-Presidente Karim Khalili), Noorin (próxima al Vicepresidente Marshal Fahim o Ayna TV (controlada indirectamente por el general Dostom, líder del Jumbesh-e-Milli) en 2004. Son cadenas pequeñas, con escasos empleados pero que encuentran público fiel a sus programas.

Las cadenas regionales y locales también han tenido un desarrollo sin precedentes desde el año 2005 y se puede decir que prácticamente cada ciudad tiene una cadena privada finaciada por un empresario local o indirectamente por personalidades políticas. En Jalalabad Sharq se fundó en el año 2008 por un empresario de la construcción propietario de tres cadenas de radio (Nargis, Sharq y Mumbar), un periódico (Shaiq) y una compañía de producción. La cadena emite dieciocho horas al día y emplea a sesenta y cuatro empleados. Arezo en Mazar-e-Sharif se estableció en 2007 por

${ }^{17}$ Shamshad $T V:$ http://eng.shamshadtv.tv/ y Lemar TV: http://www.lemar.tv/ 
el empresario Kamal Nabizade y emite principalmente videos musicales y películas (muchas de Tajikistán) y llega a tres provincias en Uzbekistán. Hewad en Kandahar inició su andadura en 2006 de la mano del empresario Qazi Mohammad Omar (a su vez propietario de la cadena de radio Afghan Azad) y emite en pastú. En Herat Taban $T V$ propiedad de Abdul Karim Sadiqi desde 2008 con una cobertura que se extiende a los ocho distritos que circunscribe la región.

\section{Programas Televisivos: de la religión al juego}

El estudio sobre el panorama televisivo en Afganistán mostraba que del año 2002 al 2005 los medios de comunicación se centraban principalmente y casi con exclusividad en la información y en el entretenimiento, dejando de lado otros temas como la educación o el debate social. Y a partir del 2005 y sobre todo desde el año 2008 aparecieron en pantalla nuevas series indias o extranjeras con temas que giran en torno a la familia y las relaciones de género, entre otros aspectos. La mayor parte de las series televisivas emitidas en Afganistán se producen en la India y se doblan al dari. Tolo TV fue la primera en emitir la popular serie: Zaman, Khushu Ham Arus Bud (Un tiempo atrás la madrastra también fue novia), un drama familiar que recibió críticas de los sectores más conservadoras al presentar temas como las relaciones extramatrimoniales. También priman las series turcas Dunya-e-Asrar (El mundo de los secretos), las series iraníes -más vistas en Herat o por la comunidad que ha vuelto de ese país a Afganistán- o incluso las de Corea del Sur y que atraen a una de las minorías de Afganistán: los hazaras. Las series de producción afgana como Eshq-e-Piri (El amor en la vejez) se emite en la cadena pública $R T A$ y presenta la vida humilde de una zona rural y Raz Ha e Een Khana (Secretos de la Casa) en Tolo $T V$ y refleja temas sociales centrados en el exilio, el retorno, el contraste entre las generaciones y los asuntos relacionados con el matrimonio. La serie se emite una vez a la semana, los viernes por la tarde y ha sido tal el éxito que paraliza la vida pública ${ }^{18}$.

Todas las cadenas emiten un programa religioso: Cheragh hedayat (El camino de la orientación) de la cadena Ariana es el que cuenta con mayor índice de audiencia. Farhang wa Tamaddon-e-Islam (Cultura y civilización del islám) en Tolo TV se centra en temas religiosos pero también otros relacionados con la arquitectura o la historia. Desde el año 2006 comenzaron los programas de tinte político como Spini Khabari en Afghan TV o Akher-e-Khat (El final de la línea) en la cadena Noor o Gofteman (Forum) de Tolo TV que reúne a cinco políticos (la mayoría miembros del Parlamento) para discutir sobre temas de sociedad. Los programas que consiguen más índice de audiencia después de las series son los de entretenimiento o juego como Zehni Azmoyono (El test de la inteligencia) en $R T A$ donde los concursantes deben adivinar diferentes preguntas, Raz-e-Taswir (Los secretos del cuadro) o Sad Dar Sad (100\%) en Ariana TV una emisión con la participación del público para resolver preguntas relacionadas con la historia, la literatura y la cultura afgana y que se ha convertido en uno de los programas

${ }^{18}$ Otras series con menor audiencia son: Nawa-e-Nay (Sonido de la Flauta) y Aseman-e-Abi (Cielo Azul) centrada en la clase trabajadora que vive en Kabul y que presenta la vida de la minoría hazaras. 
más visto en las televisones afganas. Otros programas culturales son Kakh-e-Boland (Alto Castillo) un nombre extraído del "Libro de los Reyes" la obra escrita por el poeta Ferdowsi sobre la historia de persia. El programa centrado en la literatura presenta autores de poesía clásica y moderna persa y realiza entrevistas a poetas contemporáneos afganos. Sokhan wa Andisha (Palabra y pensamiento) en RTA, toca temas relativos a la lengua y preservación del dari y pastu, literatura, cultura y civilización. Jahan-eHonar (El mundo del arte) en Azadi TV presenta entrevistas de afganos que viven en el extranjero y que han emigrado por motivos laborales pero también políticos.

Los programas de desarrollo y educativos ocupan un espacio importante tanto en las cadenas públicas como privadas. Hamsinfi (Compañero) en Tamaddon enseña ciencias a los alumnos de secundaria que optan por ir a la universidad, y en la misma cadena se emite Khabarha-e-Elimiwa Farhangi (Noticias científicas y culturales) sobre descubrimientos médicos o físicos ${ }^{19}$. Zangari Report emitido en horario prime time en la $R T A$ es el segundo programa en términos de audiencia sobre temas de desarrollo en la construcción de carreteras, hospitales ... y Rostaye Man, Khaneye Man (Mi pueblo, mi ciudad) en Azadi $T V$ viaja a una localidad en cada programa y muestra los logros alcanzados gracias a los proyectos de reconstrucción. Otros de los programas con más público son los concernientes a salud y a prevención como Dabestan Bu Ali (En la escuela de Avicena) emitido por Tolo TV, Darmalana en el que un doctor resuelve dudas a aquellos que llaman, Вапи (Dama) que se emite todas las tardes sobre aspectos relacionados con la educación, la esterilidad, o los embarazos o Zan Wa Zindagi (Mujeres y Vida) en Azadi TV sobre el papel de las mujeres y sus dificultades para alcanzar la paridad (Altai, 2010: 93).

Los programas con mayores índices de audiencia detrás de las series de televisión han sido Buenos Días Afganistán emitido desde el año $2005^{20}$. Y el concurso Afghan $S t a r^{21}$, un reality show emitido en Tolo $T V$ que busca el mejor cantante del país y copia del programa también de éxito Pop Idol en el Reino Unido o Nouvelle Star en Francia. Cada semana un grupo de jóvenes mayores de veinte años interpretaban sus canciones y los espectadores elegían a los mejores a través de sus votaciones efectuadas en los teléfonos móviles. La primera temporada tuvo lugar en septiembre de 2005 y a partir de la tercera temporada los índices de audiencia crecieron hasta once millo-

${ }^{19}$ Otro programa educativo es Gadget en Yak TV sobre innovaciones tecnológicas.

${ }^{20}$ Buenos Días Afganistán está realizado por la productora AwaNama que también realiza documentales para televisión y series como Truth and Evil Seed. Era un programa de tres horas emitido por la mañana y la tarde y realizado por periodistas locales, el éxito hizo que se emitiera en 27 de las 34 provincias afganas.

${ }^{21}$ La productora Kaboora es una de las mayores productoras de televisión. Tiene unos doscientos trabajadores en plantilla y ha creado programas de éxito como Afghan Star y la primera telenovela afgana Secrets of this House (Secretos de la Casa) ambas producidas por The Moby Group.

Otros programas centrados en el juego son Ki Mikhohad Dowlat Mand Shawad? (Quién quieres ser millonario?) en Shamshad Television, Del Na Del en Yak TV y Deal or No Deal en Tolo $T V$. Estos dos últimos programas fueron prohibidos en el año 2010 por incitar al juego y las apuestas, lo que está prohibido por el islám. 
nes de espectadores. El éxito de la serie hizo que se rodara un documental dirigido por Havana Marking que consiguió dos premios en el festival de Sundance de 2009 el World Cinema Audience Award y el World Cinema Directing Award.

\section{A modo de conclusión}

En un país en el que los medios han sido utilizados durante décadas como instrumentos de propaganda y en dónde la diseminación de la información se producía principalmente a través de discusiones "uno a uno" o explicaciones realizadas por los clérigos, las juntas de ancianos o los profesores, el papel de éstos líderes de opinión sigue siendo clave en la transmisión de la información (Grevemeyer, 1985, p. 23). El pueblo afgano muestra su desconfianza hacia los medios generalistas, a veces por ser próximos a occidente -financiadas con dinero proveniente de estos países- y otras por el descrédito con el que cuenta la clase política afgana. La comunicación que sigue funcionando es la comunicación en los lugares públicos a pequeños grupos concentrados en las mezquitas, bazares o incluso en los baños públicos (Rawan, 2002: 155-170).

El futuro de los medios de comunicación en Afganistán es tan incierto como el desarrollo del propio país. En un primer momento las donaciones de la comunidad internacional a los medios de comunicación afganos pretendían contrarrestar los efectos de las comunicaciones de los insurgentes, llegar a la población afgana y crear un sector de medios de comunicación independientes, pero la retirada progresiva de las tropas extranjeras y el desinterés de las organizaciones internacionales junto con la crisis económica mundial está teniendo un impacto directo sobre las financiación de los medios más pequeños, los más vulnerables y a los que les cuesta conseguir recursos privados, porque los anunciantes (principalmente de telefonía móvil o banca) se concentran en la televisión nacional. Al mismo tiempo los sectores más radicales han aprendido la lección sobre la importancia de la radio y de la televisión para conseguir más adeptos a su causa y están creciendo los medios locales que promueven los mensajes más radicales.

Por otra parte ser periodista en Afganistán no es tarea sencilla. Los periodistas sufren presión, autocensura e inseguridad y son numerosos los casos de torturas, secuestros y asesinatos por el hecho de informar. En enero de 2011 el joven periodista Razaq Mamoon fue agredido con ácido por sus escritos críticos con Irán y el Gobierno de Karzai. Y desde la llegada de las tropas internacionales hasta el año 2011 han sido declaradas veintidós muertes de periodistas -seis mujeres y dieciséis hombres- ${ }^{22}$ tal como estima la organización periodistas sin fronteras, aunque el número posiblemente sea mayor. Los periodistas afganos tienen dificultades para encontrar empleo y los trabajos que se ofrecen no están bien remunerados, lo que hace que la mayoría colaboren para varios medios como freelance cuando no ejercen como profesores o traductores. La $B B C$ tiene en su plantilla a treinta y cinco periodistas a tiempo completo,

22 Y aunque Afganistán goce de mayor libertad de prensa que otros países vecinos como Iran, Turkmenistan, Uzbekistan, Kyrgyzstan, Kazakhstan o incluso Pakistan, si en el año 2005 estaba en el puesto 125 de 196 países estudiados, en la actualidad está en el puesto 163. Reporteros sin Fronteras: http://www.rsf-es.org/noticias/asia/afganistan/ 
la $R T A$ emplea a ciento cuarenta periodistas y las cadenas de televisión Yak TV o Ariana tienen una plantilla de cincuenta empleados ${ }^{23}$, pero los salarios son muy variables y dependen de muchos factores entre ellos el conocimiento de idiomas. La $B B C$ es el medio que ofrece los sueldos más elevados que pueden rondar entre los mil euros al mes y los salarios menores se concentran en los medios públicos gubernamentales que no llegan a los cincuenta euros al mes.

La censura gubernamental pese a las leyes que promovían la libertad de prensa sigue creciendo. En abril de 2008 el entonces Ministro de información y Cultura Abdul Karim Khurram ordenó el cierre de Tolo $T V$ y de otras cadenas que emitían series de Bollywood por ser consideradas antiislámicas. El propietario de la cadena Saad Mohseni recurrió la sentencia y los espectadores pudieron disfrutar de estos programas. No sucedió lo mismo con Emrooz TV, una cadena televisiva con sede en Herat cerrada por decisión gubernamental en julio de 2010 por su posicionamiento en contra de la comunidad shia.

Los retos futuros son muchos e inciertos: un gobierno frágil que acoge a unos medios frágiles y que debe impulsar los programas educativos para finalizar con la alta tasa de ilestrismo. Cinco grupos mediáticos que dominan el espectro de los medios privados y que apuestan sobre todo por la televisión e internet y la cadena pública Radio Television Afghana (RTA) que subsiste gracias a las aportaciones de fondos privados (BBC, 2012). La falta de electricidad en muchas zonas (sólo el $42 \%$ de los afganos tiene acceso eléctrico), la inseguridad, el descontento y el hastío generalizado de la población tras años de luchas internas e invasiones externas hacen de este país un barco a la deriva que se refugia en los programas como Afghan Idol, porque quizá solo sea la música de lo que les ayude a soportar tanta tragedia.

\section{Referencias}

ALTAI CONSULTING (2005): Afghan Media, Three Years After: Media and Alternative Sources of Information in Afghan Society, www.atlaiconsulting.com/docs /media/2005. [fecha de consulta: 20 de agosto de 20012]

ALTAI CONSULTING (2010): Afghan Media in 2010. http://www.altaiconsulting .com/docs/media/2010/Afghan\%20Media\%20in\%202010.pdf [fecha de consulta: 15 de julio de 2012]

BBC (2012): The media of Afghanistan: The challenges of transition. Media Action PolicyBriefing. http://downloads.bbc.co.uk/mediaaction/policybriefing/bbc_media_action_afghanistan_is_in_transition.pdf [fecha de consulta: 1 de septiembre de 2012]

GREVEMEYER, Jan Heeren (1985): “Im Windschatten des Krieges: Die Entstehung eines neuen Publikationswesens im afghanischen Widerstand" [In the slipstream of war]. In Blätter des Informationszentrums Dritte Welt (pp. 128-136). Freiburg, Prolit-Verlag.

23 Kabul y Khost son las únicas universidades que cuentan con una Facultad de periodismo. La Facultad de Periodismo de Kabul se fundó en 1985 y cuenta con unos quinientos estudiantes de los el $30 \%$ son mujeres. 
GREVEMEYER, Jan Heeren (1987): Afghanistan: Sozialer Wandel und Staat im 20. Jahrhundert [Afghanistan: Social change and state in the 20th century]. Berlin, Express Edition.

GROTZBACH, Erwin (1979): Städte und Basare in Afghanistan (Ciudades y bazares en Afganistan]. Wiesbaden, Reichert.

RAWAN, Shir Mohammad (2002): "Modern Mass Media and Traditional Communication in Afghanistan". Political Communication, No. 9, pp. 155-170.

SHABIR, Ghulam; SHAHZAD, Ali; ZAFAR, Iqbal (2011): "US Mass Media and Image of Afghanistan: Portrayal of Afghanistan by Newsweek and Time", South Asian Studies. Vol. 26, No. 1, pp. 83-101.

UNESCO (2012): Comunication and Information Afganistán. http://www.unesco .org/new/en/communication-and-information/freedom-of-expression/professionaljournalistic-standards-and-code-of-ethics/south-asia. [fecha de consulta: 29 de agosto de 2012]

YUSSUFI, Nadjib (1977): Publizistik der Entwicklungsländer am Beispiel Afghanistans [El periodismo en los países en desarrollo]. Unpublished doctoral dissertation, University of Berlin, Germany.

\subsection{Webs consultadas por orden de aparición en el artículo}

Internews: http://www.internews.org/. [fecha de consulta: 15 de julio de 2012]

Institute for War \& Peace Reporting (IWPR) http://iwpr.net/ [fecha de consulta: 20 de junio de 2012]

Ministerio de Información y Cultura (MoIC): http://moic.gov.af/en [fecha de consulta: 5 de mayo de 2012]

Kabul Weekly: www.kw.af. [fecha de consulta: 3 de mayo de 2012]

Pajhwok Afghan News (PAN), http://www.pajhwok.com/ [fecha de consulta: 18 de julio de 2012].

Benawa: www.benawa.com [fecha de consulta: 8 de mayo de 2012].

Payam-aftab: www.payam-aftab.com [fecha de consulta: 20 de octubre de 2012].

Kabul Press: www.kabulpress.org [fecha de consulta: 17 de noviembre de 2012]

Ariana TV: http://arianatelevision.com/ [fecha de consulta: 2 de mayo de 2012].

Tolo TV: http://tolo.tv/ [fecha de consulta: 27 de mayo de 2012]

Shamshad TV: http://eng.shamshadtv.tv/ [fecha de consulta: 30 de mayo de 2012]

Lemar TV: http://www.lemar.tv/ [fecha de consulta: 30 de junio de 2012]

Reporteros sin Fronteras: http://www.rsf-es.org/noticias/asia/afganistan/ [fecha de consulta: 17 de mayo de 2012] 